\title{
https://doi.org/10.46813/2021-136-072 ACCELERATION OF ELECTRONS USING INVERSE ANOMALOUS SCATTERING
}

\author{
V.V. Ognivenko \\ National Science Center “Kharkov Institute of Physics and Technology”, Kharkiv, Ukraine \\ E-mail:ognivenko@kipt.kharkov.ua
}

Acceleration of electrons in the field of two electromagnetic waves propagating in a slowing down medium in the direction of motion of electrons is considered under conditions when one of the waves propagates with a phase velocity greater than the velocity of electrons, and the other with a phase velocity less than the velocity of electrons. Taking into account deceleration by radiation, we determine dependence of the electron energy on the coordinate along the direction of acceleration. Thus, we obtain the expression for the maximum electron energy and effective acceleration length.

PACS: 41.60.-m, 41.75.Jv, 41.20.Jb

\section{INTRODUCTION}

A number of new methods of charged particles acceleration are known to use the inversion of the elementary effects of radiation and scattering of electromagnetic waves by moving charged particles [1]. Studying the possibility of inverting the known mechanisms of electromagnetic waves emission is important for the search of new methods of the charged particles acceleration $[2,3]$. In this work, we investigated the possibility of accelerating electrons under conditions of the inverse anomalous light scattering effect $[4,5]$. The author analyzed the mechanism of charged particles acceleration, as well as the physical reasons limiting the increase in the energy of electrons.

\section{THE KINEMATICS OF SCATTERING}

The elementary effect of anomalous scattering is that an incident photon with a frequency of $\omega_{1}$, scattered by a relativistic electron moving in a refractive medium, causes the appearance of an induced photon of the same frequency $\omega_{1}$ and a photon of scattered light with a frequency of $\omega_{2}$ [4]. The laws of energy conservation and momentum of an electron in an elementary act of anomalous scattering can be written in the form:

$$
\varepsilon_{2}=\varepsilon_{1}-\hbar \omega_{1}-\hbar \omega_{2}, \mathbf{p}_{2}=\mathbf{p}_{1}-\hbar \mathbf{k}_{1}-\hbar \mathbf{k}_{2},
$$

here $\varepsilon_{1}, \mathbf{p}_{1}$ and $\varepsilon_{2}, \mathbf{p}_{2}$ are the energy and momentum of an electron before and after interaction with photons, $\hbar \omega_{i}$ and $\hbar \mathbf{k}_{i}$ are the energy and momentum of a photon, $i=1,2$.

In the elementary process of inverse anomalous scattering, the energy and momentum of two incident photons of frequencies $\omega_{1}$ and $\omega_{2}$ are given to a particle. In this case, the laws of conservation of energy and momentum have the form

$$
\varepsilon_{2}=\varepsilon_{1}+\hbar \omega_{1}+\hbar \omega_{2}, \mathbf{p}_{2}=\mathbf{p}_{1}+\hbar \mathbf{k}_{1}+\hbar \mathbf{k}_{2} .
$$

Assuming that the momentum of a photon in a medium is equal $\frac{\hbar \omega n}{c} \frac{\mathbf{k}}{|\mathbf{k}|}$, and the energy of photons is small compared to the energy of a particle, it follows from (2) that frequencies $\omega_{1}$ and $\omega_{2}$ satisfy the relation

$$
\omega_{2}=\omega_{1} \frac{\beta_{0} n\left(\omega_{1}\right) \cos \theta_{1}-1}{1-\beta_{0} n\left(\omega_{2}\right) \cos \theta_{2}},
$$

similar to the case of anomalous scattering of light by a moving charged particle (1). Here and in what follows, for simplicity, we assume that the medium is isotropic, characterized by a refractive index $n(\omega)$ that depends on the frequency, $\boldsymbol{\beta}=\mathbf{v} / c, \theta_{1}$ and $\theta_{2}$ are the angles between the particle velocity $\mathrm{v}$ and the wave vectors $\mathbf{k}_{1}$ and $\mathbf{k}_{2}$, and $c$ is the speed of light in vacuum.

It should be noted that the stimulated anomalous scattering of an electromagnetic wave by an intense relativistic electron beam leads to the development of collective beam instability, as a result of which the intensities of the incident and scattered radiation increase simultaneously. The condition for the resonance of the beam electrons with the incident and scattered electromagnetic waves has the form [6]

$$
\omega_{1}+\omega_{2}=\mathbf{v}\left(\mathbf{k}_{1}+\mathbf{k}_{2}\right) \text {. }
$$

In the case of anomalous scattering and the corresponding inverse effect, it is necessary that for one wave the condition is satisfied $\beta n_{1} \cos \theta_{1}>1$, while for the other wave the condition is $\beta n_{2} \cos \theta_{2}<1$. Here $\mathrm{n}_{\mathrm{i}}=\mathrm{n}\left(\omega_{\mathrm{i}}\right)$. Below we will consider the process inverse to stimulated anomalous scattering, namely, the acceleration of electrons in the field of two incident electromagnetic waves of frequencies $\omega_{1}$ and $\omega_{2}$, when the waveresonance condition (4) is satisfied.

\section{ACCELERATION DYNAMICS}

Let an electron flow move in the positive direction of the $\mathrm{z}$ axis with an initial velocity $\mathrm{v}_{0}$. Two plane monochromatic waves $\mathbf{E}_{1}$ and $\mathbf{E}_{2}$ propagate in the same direction:

$$
\mathbf{E}_{1}(z, t)=\mathbf{e}_{x} A_{1} \sin \varphi_{1}, \mathbf{E}_{2}(z, t)=\mathbf{e}_{x} A_{2} \sin \varphi_{2},
$$

where $\mathbf{A}_{\mathbf{i}}$ is the amplitude of the wave, $\varphi_{i}=k_{i} z-\omega_{i} t$, $\omega_{\mathrm{i}}, \mathrm{k}_{\mathrm{i}}$ are the frequencies and wave vectors of the waves, $i=1,2 ; \mathbf{e}_{\mathrm{x}}$ is a unit vector along the x-axis. Let $\omega_{2}>\omega_{1}$ and the phase velocity of one of the waves is less than the velocity of the beam, and the phase velocity of other wave is greater than its velocity:

$$
\mathbf{v}_{p h 1}=\frac{\omega_{1}}{k_{1}}<c, \mathbf{v}_{p h 2}=\frac{\omega_{2}}{k_{2}} \geq c .
$$

The electromagnetic fields (5) produce a transverse oscillator speed of electrons. Taking into account the conservation of the generalized transverse (perpendicu- 
lar to the $\mathrm{z}$-axis) momentum of the electrons in the electromagnetic fields (5), the expression for the transverse oscillator velocity of the electrons can be written in the form

$$
\mathbf{v}_{\perp}=\mathbf{e}_{x} \frac{c}{\gamma}\left(a_{1} \cos \varphi_{1}+a_{2} \cos \varphi_{2}\right)
$$

where $a_{i}=\frac{e A_{i}}{m c \omega_{i}}, \gamma=\left(1-\boldsymbol{\beta}^{2}\right)^{-1 / 2}$ is the relativistic

factor, $e$ is the electron charge, $m$ is its mass.

In the longitudinal direction, along the $\mathrm{z}$ axis, the electrons move under the action of the ponderomotive force generated by the beats of the two considered electromagnetic waves. The dynamics of changes in the energy of electrons in the field of these waves is described by the equation:

$$
\frac{d \varepsilon}{d t}=e \mathbf{v}\left(\mathbf{E}_{1}+\mathbf{E}_{2}\right)+\left(\frac{d \varepsilon}{d t}\right)_{R}
$$

where $\varepsilon=m c^{2} \gamma$ is the energy of an electron.

The second term in equation (8) describes the radiation energy loss of an individual electron for radiation.

$$
\begin{aligned}
& \left(\frac{d \varepsilon}{d t}\right)_{R}=-\frac{e^{2}}{3 c \gamma^{2}}\left[n_{2} \omega_{1}^{2} a_{1}^{2} \frac{\left(\beta n_{1}-1\right)^{2}}{\left(1-\beta^{2} n_{2}^{2}\right)^{2}}+\right. \\
& \left.+n_{1} \omega_{2}^{2} a_{2}^{2} \frac{\left(1-\beta n_{2}\right)^{2}}{\left(\beta^{2} n_{1}^{2}-1\right)^{2}}\right] .
\end{aligned}
$$

The first term in the eq. (9) is due to the loss of the electron energy for radiation in the field of the electromagnetic wave $\mathbf{E}_{1}$, and the second term in this equation is due to the loss of energy of an electron for radiation in the field of the electromagnetic wave $\mathbf{E}_{2}$.

Substituting expressions (5), (7) and (9) in (8) and keeping the resonance terms $\mathrm{v}_{z} \approx \omega_{3} / k_{3}$, for relativistic electrons $\left(\gamma^{2}>>1\right)$ we obtain the following equations of motion:

$$
\begin{gathered}
\frac{d \gamma}{d z}=\frac{\omega_{3}}{2 \mathrm{v}_{z} \gamma} a_{1} a_{2} \sin \psi-\frac{1}{3} r_{0} \sigma^{2} \gamma^{2}, \\
\frac{d \psi}{d z}=k_{3}-\frac{\omega_{3}}{c}-\frac{\omega_{3}}{2 c \gamma^{2}}\left(1+\frac{a_{1}^{2}}{2}+\frac{a_{2}^{2}}{2}\right),
\end{gathered}
$$

where $\quad \sigma=\frac{\omega_{1}}{c}\left(\beta n_{1}-1\right) a_{1}, \quad r_{0}=\frac{e^{2}}{m c^{2}}, \quad n_{i}=\frac{c}{\mathrm{v}_{p h i}}$ $\psi=k_{3} z-\omega_{3} t, \quad \omega_{3}=\omega_{1}+\omega_{2}, k_{3}=k_{1}+k_{2}$. Here, instead of $t$, we introduced a new independent variable $z \geq 0$. When calculating the radiation loss of an individual electron in the eq. (10), it was assumed that the frequency $\omega_{2}$ is so high that $\mathrm{n}_{2}=1$, and also that $a_{1,2}<1$, $a_{2} \ll a_{1} \gamma^{2}\left(\beta^{2} n_{1}^{2}-1\right) / n_{1}$.

Consider the motion of an equilibrium (synchronous) particle, whose phase relative to the combination wave remains constant during the acceleration process: $\psi_{\mathrm{c}}=$ const. Then, from Eq. (10) with a fixed value of the parameter $\sigma$, we obtain the following equation, which determines the energy of an equilibrium particle $\gamma_{c}$ as an implicit function of the coordinate $\mathrm{z}$ :

$$
\frac{\kappa z}{\gamma_{*}^{2}}=\Phi\left(\frac{\gamma_{c}}{\gamma_{*}}\right)-\Phi\left(\frac{\gamma_{0}}{\gamma_{*}}\right)
$$

where

$\Phi(x)=\int \frac{x d x}{1-x^{3}}=\frac{1}{6} \ln \left[\frac{x^{2}+x+1}{(1-x)^{2}}\right]-\frac{1}{\sqrt{3}} \operatorname{arctg}\left(\frac{2 x+1}{\sqrt{3}}\right)$, $\gamma_{*}=\left(\frac{3 c \omega_{3}}{2 r_{0} \omega_{1}^{2}} \frac{a_{2}}{a_{1}} \frac{\sin \psi_{c}}{\left(\beta_{c} n_{1}-1\right)^{2}}\right)^{1 / 3}, \quad \kappa=\frac{\omega_{3}}{2 c} a_{1} a_{2} \sin \psi_{c}$, $\gamma_{0}=\gamma_{c}(0)$, the subscript "c" denotes the values of the equilibrium particle.

It follows from equations (10) and (12) that the energy of electrons in the accelerating phase $\sin \psi_{c}>0$ increases as they move along $\mathrm{z}$.

In the case $\gamma_{c}<<\gamma_{*}$, when the ponderomotive force accelerating the electrons significantly exceeds the force of radiative deceleration of an individual electron, from Eq. (12) we obtain the following law of increasing the energy of an equilibrium particle

$$
\gamma_{c}=\sqrt{\gamma_{0}^{2}+2 \kappa z} \text {. }
$$

In the case $\gamma_{*}-\gamma_{c}<<\gamma_{*}$, when the ponderomotive force accelerating the electrons significantly exceeds the damping force by the radiation of an individual electron, from Eq. (12) we obtain the following law for the increase in the energy of an equilibrium particle:

$$
\gamma_{c}(z)=\gamma_{*}-\left(\gamma_{*}-\gamma_{0}\right) B\left(\frac{\gamma_{0}}{\gamma_{*}}\right) \exp \left(-\frac{3 \kappa z}{\gamma_{*}^{2}}\right),
$$

where

$$
B(x)=\frac{1}{\sqrt{1+x+x^{2}}} \exp \left[3 \operatorname{arctg}\left(\frac{1+2 x}{\sqrt{3}}\right)-\pi\right] .
$$

Let's note that the function $B(x)$ monotonically increases from a value approximately equal to 0.7 at $x<<1$ to unity at $x \rightarrow 1$.

Equation (14) shows that the limiting energy to which electrons can be accelerated is

$$
\varepsilon_{*}=m c^{2} \gamma_{*}=m c^{2}\left(\frac{3 c \omega_{3}}{2 r_{0} \omega_{1}^{2}} \frac{a_{2}}{a_{1}} \frac{\sin \psi_{c}}{\left(\beta_{c} n_{1}-1\right)^{2}}\right)^{1 / 3} .
$$

$\gamma_{c} / \gamma_{\star}$

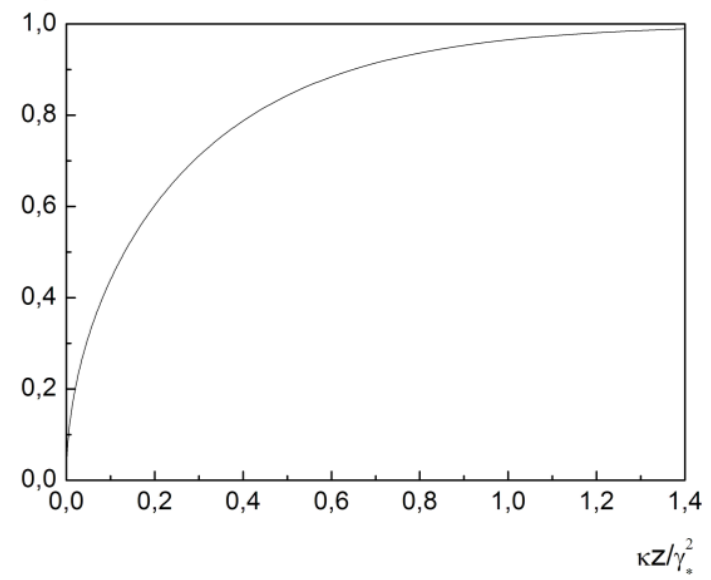

Dependence of the normalized particle energy on the normalized acceleration length 
Figure shows the graph obtained by solving equation (12). As can be seen from Figure at $z \approx \gamma_{*}^{2} / \kappa$, the particle energy approaches the maximum energy (the difference is about 3\%). For relativistic particles, taking into account that the wave frequencies satisfy the relations (3) and (6), the expression (15) can be written in the form

$$
\varepsilon_{*}=m c^{2}\left(3 \frac{\lambda_{1 \mathrm{v}}}{r_{0}} \gamma_{0}^{2} \frac{a_{2}}{a_{1}} \frac{\sin \psi_{c}}{\beta_{c} n_{1}-1}\right)^{1 / 3},
$$

where $\lambda_{1 \mathrm{v}}=c / \omega_{1}$.

From this equation it follows, for example, that for electrons with an initial energy of tens of megaelectronvolt, an electromagnetic wave $E_{1}$ in the centimeter range and parameters $a_{2}>a_{1}$, maximum energy of accelerated electrons can be several tens of gigaelectronvolt. The limitation of the maximum electron energy in the considered acceleration method is due to the fact that at values of $\gamma_{c}$ close to $\gamma_{*}$, practically all the energy acquired by the particle in the accelerating field of the combination wave is lost by the particle to radiation.

Equation (14) and Fig. 1 also show that the characteristic distance $\mathrm{L}_{*}$, at which electrons gain energy close to the maximum possible, is equal to

$$
L_{\bullet \mathrm{p}}=\frac{\gamma_{*}^{2}}{3 \kappa} \text {. }
$$

We note that this distance increases with an increase in the maximum electron energy and the acceleration length up to energies of several gigaelectronvolt, in the considered example, can be hundreds of meters.

\section{CONCLUSIONS}

Continuous acceleration in the considered case is possible while maintaining the synchronism of electrons with the combination wave along the acceleration length. For this, one can, for example, change the geometry of the slow wave structure or alternate sections with different values of frequencies $\omega_{1}$ and $\omega_{2}$.
Since the accelerating force is proportional to the square of the charge, electrons and positrons can be accelerated in one bunch, compensating the space charge of each other.

It should also be noted that the above calculations of the dynamics of electron acceleration correspond to the case when the ionization losses by particles are small. This can be achieved, for example, when accelerating in plasma in the field of intense laser radiation and a slow plasma wave (plasma FEL $[6,7]$ ) or upon acceleration in a thin channel in the medium. In the latter case, the radius of the channel should be less than the wavelength of the low-frequency slow wave and may be much larger than the wavelength of the high-frequency (laser) radiation.

\section{REFERENCES}

1. YA.B. Faynberg. Nekotoryye voprosy plazmennoy elektroniki // Fizika plazmy. 1985, v. 11, № 11, p. 1398-1410 (in Russian).

2. A.N. Lebedev, A.V. Shal'nov. Osnovy fiziki $i$ tekhniki uskoriteley. M.: "Energoatomizdat", 1991, 528 p. (in Russian).

3. V.V. Ognivenko. Uskoreniye elektronov s pomoshch'yu obrashchennogo anomal'nogo rasseyaniya // DAN Ukrainy. 2000, № 9, p. 91-94 (in Russian).

4. I.M. Frank. Rasseyaniye sveta elektronom, dvizhushchimsya $\mathrm{v}$ prelomlyayushchey srede // Yadernaya fizika. 1968, v. 7, № 5, p. 1100-1105 (in Russian).

5. I.M. Frank. Eynshteyn i optika // UFN. 1979, v. 129, № 4, p. 685-703 (in Russian).

6. V.V. Ognivenko. Vynuzhdennoye rasseyaniye elektromagnitnoy volny relyativistskim elektronnym puchkom v srede: Prepr. / AN USSR. KHFTI; 83-42. Khar'kov. 1983, 22 p. (in Russian).

7. YA.B. Faynberg. Uskoreniye zaryazhennykh chastits v plazme // Fizika plazmy. 1997, v. 23, № 4, p. 275-283 (in Russian).

Article received 04.10.2021

\section{УСКОРЕНИЕ ЭЛЕКТРОНОВ С ПОМОЩЬЮ ОБРАЩЕННОГО АНОМАЛЬНОГО РАССЕЯНИЯ}

\section{В.В. Огнивенко}

Рассмотрено ускорение электронов в поле двух электромагнитных волн, распространяющихся в замедляющей среде в направлении движения электронов, когда одна из волн распространяется с фазовой скоростью большей скорости электронов, а другая с фазовой скоростью меньшей скорости электронов. Учитывая торможение излучением, определена зависимость энергии электронов от длины ускорения. Получено выражение для максимальной энергии электронов и эффективной длины ускорения.

\section{ПРИСКОРЕННЯ ЕЛЕКТРОНІВ ЗА ДОПОМОГОЮ ОБЕРНЕНОГО АНОМАЛЬНОГО РОЗСІЯННЯ}

\section{В.В. Огнівенко}

Розглянуто прискорення електронів у полі двох електромагнітних хвиль, що поширюються в уповільнюючому середовищі в напрямку руху електронів, коли одна із хвиль поширюється з фазовою швидкістю більшою швидкості електронів, а інша $з$ фазовою швидкістю меншою швидкості електронів. Враховуючи гальмування випромінюванням, визначена залежність енергії електронів від координати уздовж напрямку прискорення. Отримано вираз для максимальної енергії електронів і ефективної довжини прискорення. 